\title{
Enduring Positivity: Children Of Incarcerated Parents Report More Positive Than Negative Emotions When Thinking about Close Others
}

\author{
James P. Dunlea (D), Redeate G. Wolle, and Larisa Heiphetz \\ Columbia University
}

\begin{abstract}
Millions of children in the United States experience parental incarceration, yet it is unclear how this experience might shape social cognition. We asked children of incarcerated parents $(N=24)$ and children whose parents were not incarcerated $(N=58)$ to describe their parents. Both groups of children also rated the extent to which they agree that they feel positive and, separately, negative emotions when thinking about their parent and best friend. This approach allowed us to test between two alternative hypotheses. On the one hand, cultural narratives in the United States convey negative messages about incarcerated people, and these messages could prompt children to report negativity when thinking about their incarcerated parents. On the other hand, children's positivity toward close others is robust. Thus, when thinking about their incarcerated parents, children may report a great deal of positivity. Consistent with the latter possibility, children were more likely to describe their incarcerated parents using positive rather than negative terms. Moreover, children of incarcerated parents were more likely to agree that thinking about close others made them feel positive emotions than they were to agree that thinking about close others made them feel negative emotions. A similar pattern of results emerged among children whose parents were not incarcerated. These findings demonstrate the robustness of children's positivity and can inform debates regarding contact between incarcerated parents and their children.
\end{abstract}

The United States has a higher incarceration rate than any other country (Sawyer \& Wagner, 2020). Thus, it is hardly surprising that incarceration affects many people who have never been imprisoned themselves, including children, at least five million of whom have experienced parental incarceration in their lifetime (Murphey \& Cooper, 2015). While much work has emphasized the role of parental incarceration in shaping outcomes in domains such as health and education (Arditti, 2012; Murray \& Farrington, 2008), relatively less work has examined how parental incarceration may shape social cognitive processes such as person perception. The present work addressed this topic by asking children of incarcerated parents as well as children whose parents were not incarcerated about their perceptions of - and emotions when thinking about - individuals in two different types of close relationships, namely, their parent and their best friend.

CONTACT James P. Dunlea james.dunlea@columbia.edu Department of Psychology, Columbia University, New York, NY 10027

(4) Supplementary data for this article can be accessed on the publisher's website. 
Past work leads to two competing predictions regarding the role of parental incarceration in shaping children's views of close others. On the one hand, children may report negativity when thinking about their incarcerated parents. Predominant cultural messages in the United States portray incarcerated individuals as having committed severe moral transgressions (Kleinfeld, 2016), and such messages could influence children's perspectives (e.g., Birnbaum, Deeb, Segall, Ben-Eliyahu, \& Diesendruck, 2010; Diesendruck \& Haber, 2009; Gelman, 2009). Indeed, when thinking about why individuals come in contact with the justice system, both children of incarcerated and non-incarcerated parents readily attribute such contact to individuals' negative internal qualities, such as their bad moral character or immoral desires (Dunlea \& Heiphetz, 2020). Further, children often feel negatively toward transgressors, especially those who commit moral transgressions (Hardecker, Schmidt, Roden, \& Tomasello, 2016). Together, societal messages and their own sensitivity to perceived moral violations may lead children to express negativity when thinking about their incarcerated parents.

On the other hand, children may report positivity when thinking about their incarcerated parents. This possibility is consistent with work suggesting that children's positivity toward others is robust (e.g., Boseovski \& Lee, 2006; Diesendruck \& Lindenbaum, 2009). In one line of work investigating whether children are more likely to infer character traits from positive or negative actions, children received either one or five pieces of information about an actor's past behavior and subsequently made trait attributions about that person (Boseovski \& Lee, 2006). Overall, children required fewer pieces of evidence before making positive rather than negative character attributions, suggesting that children may be reticent to report negative views of other individuals even if such individuals have previously transgressed. Work investigating children's coping and resilience also supports this possibility. Children sometimes manage challenges by focusing on positive aspects of a negative situation (Zimmer-Gembeck \& Skinner, 2011). While little research has examined coping among children of incarcerated parents (for exceptions, see Johnson \& Easterling, 2015; Nesmith \& Ruhland, 2008), it is possible that they use similar strategies. Therefore, when thinking about their incarcerated parents, children may be more likely to think of positive versus negative characteristics.

In addition to investigating how parental incarceration shapes children's views of their parents, we examined the extent to which parental incarceration shapes children's views of individuals in different types of close relationships, namely, best friends. If, for instance, we found that children of incarcerated parents were more likely than children of nonincarcerated parents to agree that thinking about their parents made them feel negative emotions, we would be unable to conclude whether this effect is specific to parents or whether it generalizes to other types of close relationships. Asking about two types of close relationships allowed us to better understand whether the link between parental incarceration and social cognition is domain-specific, arising only in response to questions about an incarcerated parent, or domain-general, arising in response to questions about other significant relationships as well. The current work asked children about a best friend because children form close relationships with best friends and rate these relationships as more caring, intimate, and exclusive than other friendships (Cleary, Ray, LoBello, \& Zachar, 2002).

Some work suggests that the link between parental incarceration and social cognition may be domain-general. People who have experienced adversity in childhood are more 
likely than others to view the world - and those who inhabit it - in a negative light (JanoffBulman, 1989). Because parental incarceration is often conceptualized as a type of adversity (Arditti, 2012), this experience may impact children's views of parents and best friends. However, a separate body of work suggests that the link between parental incarceration and social cognition may be domain-specific. Children differentiate between those who transgress and those who do not (e.g., Yang, Choi, Misch, Yang, \& Dunham, 2018). This differentiation is so stark that children's initial preference for non-transgressors over transgressors persists over time (Tasimi \& Johnson, 2019). Taken together, this work suggests that children may view their incarcerated parent more negatively than their best friend.

In addition to testing between these possibilities, the current work clarifies how early systems of moral cognition constrain later socio-moral judgments. Past work suggests that adults' positivity toward close others may supersede the negativity typically associated with perceived transgression. Adults anticipate protecting close others who commit moral transgressions (Weidman, Sowden, Berg, \& Kross, 2019) and rate behaviors as less unethical when the target is a relative versus a stranger (Lee \& Holyoak, 2020). If children report more positive than negative views of their incarcerated parents, this would suggest that adults' propensity to show positivity toward close others who have transgressed may not depend on extensive social experience. Thus, in conjunction with past work testing adults, the present work provides insight into when relational closeness begins to shape moral cognition.

Examining reports of how children of incarcerated parents feel when thinking about close others also has practical importance. Positive interpersonal emotions are generally linked with healthy development (Gerhardt, 2004) and can help individuals "bounce back" from negative experiences (Tugade \& Fredrickson, 2004). However, chronic negative interpersonal emotions often disrupt healthy attachment in close relationships (e.g., Cassidy, 1994). Given the link between emotions and life outcomes, elucidating children's emotions when thinking about close others may help inform future interventions aimed at promoting positive developmental trajectories. Children of incarcerated parents are especially likely to experience negative outcomes stemming from societal inequality (Miller, 2006), yet psychological science has not focused on understanding their experiences. Illustrating the exclusion of this group, parental incarceration is not included on a list of diversity-related topics often studied by psychologists over the past decade (Blaine \& Brenchley, 2018). Thus, it is particularly important for research to take notice of this population.

In summary, the current work investigated the extent to which parental incarceration shapes children's social cognition. We asked children of incarcerated parents and children whose parents were not incarcerated to describe their parents. Additionally, children rated the extent to which they agreed that they feel positive and, separately, negative emotions when thinking about their parent and best friend. By soliciting responses from both groups of children, the present work clarifies the role of parental incarceration in social perception. 


\section{Method}

\section{Participants}

We recruited 6- to 12-year-olds with an incarcerated parent and children in the same age range whose parents were not incarcerated. Testing 6- to 12-year-olds was important for two main reasons. First, testing children in this age range allowed us to compare our results with work suggesting that children's positivity wanes throughout the elementary school years (Boseovski \& Lee, 2006). Based on this prior work, it was possible that younger children would show more positive evaluations in the current work than would older children. However, participant age did not reliably predict responses in our data (see Results section below), suggesting that positivity in the context of close relationships may be relatively more stable than positivity in other social contexts. Additionally, testing children within this age range was pragmatic: the majority of children with incarcerated parents are younger than 10 years old, and the average age of these children is eight years old (Mumola, 2000). Thus, recruiting 6- to 12-year-olds allowed us to report on the experiences of a broader segment of the population of children with incarcerated parents than if we just tested relatively younger or older children.

Our sample included 24 children of incarcerated parents $\left(M_{\text {age }}=9.38\right.$ years, $S D_{\text {age }}$ $=1.95$ years; $46 \%$ female; $4 \%$ White or European-American, $58 \%$ Black or AfricanAmerican, 13\% multiracial, 25\% other; $44 \%$ Hispanic or Latina/o) whom we recruited by partnering with two organizations that provide services to families of incarcerated individuals. One child was interviewed but excluded from subsequent analyses because she did not understand the questions. We received funding to assist with this project for one year, and we determined in advance to recruit as many participants as possible during that time period. This approach yielded a final sample size that is comparable to prior work in social and developmental psychology recruiting difficult-to-reach populations (e.g., children of incarcerated parents, Johnson \& Easterling, 2015; transgender children, Olson, Key, \& Eaton, 2015).

Twenty-nine percent of the children in this sample had an incarcerated mother, and 67\% had an incarcerated father; one child's demographic questionnaire did not indicate the gender of the incarcerated parent. Zero children had two incarcerated parents. Thirty-eight percent of children in this sample had an imprisoned parent and $38 \%$ percent had a jailed parent; six children's demographic questionnaires did not indicate whether the incarcerated parent was serving time in prison or jail. On average, children had been separated from their parent for 52.64 months $(S D=31.78$ months, range $=8-95$ months $)$ and had spoken with their parent in person or via technology (e.g., phone) an average of 14.75 times over the past month $(S D=12.37$ times, range $=1-31$ times $)$. Families with an incarcerated parent received a $\$ 20$ USD gift card for participating.

We also recruited a group of children whose parents were not incarcerated. Based on recommendations to include approximately 50 participants per cell in psychological research (Lakens \& Evers, 2014; Simmons, Nelson, \& Simonsohn, 2013), we aimed to recruit approximately 50 participants in this comparison group. We slightly overrecruited because we expected some data to be unusable. The final sample included 58 children $\left(M_{\mathrm{age}}=8.11\right.$ years, $S D_{\mathrm{age}}=1.40$ years; $69 \%$ female; $37 \%$ White or EuropeanAmerican, 30\% Black or African-American, 4\% Asian or Asian-American, 2\% Native American or Pacific Islander, 13\% multiracial, 15\% other, remainder unspecified; 33\% 
Hispanic or Latina/o). Four additional children were interviewed but excluded from subsequent analyses because a parent interfered during the interview $(n=1)$, the child did not understand the questions $(n=2)$, or the child did not speak English $(n=1)$. Additionally, one child completed the study twice; analyses only included his responses from the first session. Children were recruited from a departmental database and a children's museum in a large city in the northeastern United States; all children received a small prize for participating.

The current samples differed in ways that reflect the demographics of individuals involved in the criminal justice system. Namely, the proportion of White participants was greater in the sample of children whose parents were not incarcerated than in the sample of children of incarcerated parents. Moreover, the proportion of Black participants was greater in the sample of children whose parents were incarcerated than in the sample of children whose parents were not incarcerated. The current samples also differed in ways that do not necessarily reflect meaningful theoretical differences pertinent to the criminal justice system. Namely, the proportion of female children was greater in the sample of children whose parents were not incarcerated than in the sample of children of incarcerated parents. Additionally, the sample of children with incarcerated parents was, on average, older than the sample of children whose parents were not incarcerated; however, age did not reliably predict participant responses (see Results section below). The samples were similar on other demographic variables, such as reported ethnicity. .

\section{Procedure}

Children of incarcerated parents completed the session in a quiet room located in our partnering organizations' office space or in a developmental psychology laboratory. Children of parents who were not incarcerated participated in a quiet room in a children's museum or in a developmental psychology laboratory. First, the interviewer said that he or she would ask the child questions and that there were no right or wrong answers. The interviewer specified that some questions would be about the child's parent and others would be about other people. The interviewer then said, "First, I'm curious to learn a little more about your [mom/dad]. What is [she/he] like?"1 Children of incarcerated parents answered about their incarcerated parent. The remaining children answered about a parent who was not present during testing to help better match the conditions in which children of incarcerated parents were answering questions (i.e., both groups of children answered about a parent who was physically absent).

The purpose of this open-ended question was to determine how participants might spontaneously describe their parent when not guided by the interviewer. This approach allowed us to determine what types of traits and qualities are conceptually central to children's representations of their parents without imposing our own views (Cimpian \& Markman, 2009; Rhodes, 2014). One potential drawback, however, is that open-ended questions may require more verbal and cognitive ability than closed-ended items. Consequently, open-ended items may not fully capture children's thoughts regarding complex topics (e.g., emotions, Ganea, Lillard, \& Turkheimer, 2004). To address the

\footnotetext{
${ }^{1}$ These questions were part of a larger project probing children's understanding of punishment and the criminal justice system (Dunlea \& Heiphetz, 2020).
} 
possibility that children may not articulate all of their thoughts, we used closed-ended questions to complement the open-ended items. As well as possibly helping children communicate their thoughts about emotion, closed-ended items allowed us to use a widelyimplemented, previously validated scale measuring positive and negative affect (Watson, Clark, \& Tellegen, 1988). Though it was possible that differences in cognitive or linguistic ability might elicit different patterns of responding among younger versus older children, we did not find evidence that age predicted participants' responses to either the open- or closed-ended items (see Results section below). This suggests that even younger children did not have difficulty answering either type of question.

The interviewer then introduced children to a five-point Likert-type scale consisting of stick figures arranged from smallest to largest on a sheet of paper and instructed children on how to use the scale (e.g., asking them to point to the smallest picture if they didn't agree at all with a sentence the interviewer said). The remaining labels were "agree a little bit," "agree a medium amount," "agree a lot," and "agree completely." We scored participants' responses numerically, such that greater agreement corresponded with higher numerical values on the Likert-type scale. Numerical values ranged from 1 (don't agree at all) to 5 (agree completely). The interviewer asked children to rate their agreement with two sentences to gauge their understanding of the scale ("Right now, we're in the United States of America" and "Christmas is in the summer"). On average, children used the scale correctly: 95\% correctly agreed that they were in the United States and 96\% correctly disagreed that Christmas is in the summer. The main patterns of results reported below holds even after excluding participants who incorrectly responded to one or both of the scale comprehension check questions.

Following these instructions, children rated the extent to which they agreed that thinking about close others made them feel positive and, separately, negative emotions. Children's reported agreement served as the primary dependent variable. In one block, participants rated the extent to which thinking about their parent made them feel certain emotions (e.g., "How much do you agree that thinking about your parent makes you feel angry?"). In another block, participants rated the extent to which thinking about their best friend made them feel those same emotions. As previously mentioned in the Introduction, the purpose of asking children about both their parent and best friend was to clarify the extent to which children report feeling different emotions when thinking about individuals in different types of close relationships. We confirmed that participants were thinking about a specific best friend before proceeding with the relevant questions.

Each block included five positive emotions (happy, proud, loved, excited, calm) and four negative emotions (scared, sad, angry, nervous) toward the same person. Each emotion was drawn from the Positive and Negative Affect Schedule, a scale used to measure positive and negative emotions (Watson et al., 1988). Participants answered all questions about one person before moving on to questions about the next person. The order in which participants answered questions about their parent or best friend and the order in which emotions were presented were counterbalanced across participants. Though parental incarceration is sometimes a difficult topic for children to discuss (e.g., Johnson \& Easterling, 2015), all children of incarcerated parents readily answered questions about their parent. Additionally, neither children of incarcerated parents nor children whose parents were not incarcerated experienced difficulty answering questions about their best friend. Please 
see Appendix A for research materials and Appendix B for the scale used to elicit responses to closed-ended items.

\section{Coding for open-ended descriptions of parents}

Given our interest in children's perceptions of their parents, we coded responses to the open-ended question using categories based on theoretical interest. Namely, we examined whether participants spontaneously provided positive, negative, and neutral descriptions of their parents. We categorized descriptions as "positive" if they included attributions of positively valenced traits or qualities (e.g., "he's really nice", "a sweet angel") or if they mentioned positively valenced behaviors directed toward the participant (e.g., "he cares for me", "he used to give me ice cream and take me to the park"). Similarly, we categorized descriptions as "negative" if they included attributions of negatively valenced traits or qualities (e.g., "angry, temperamental") or if they mentioned negatively valenced behaviors directed toward the participant (e.g., "she's mean when she has to be"). Finally, we categorized descriptions as "neutral" if they included attributions of traits, qualities, or behaviors that are not clearly positive or negative (e.g., "he is serious", "short hair, very tall") or if they mentioned parents" preferences (e.g., "likes watching TV", "likes the color blue").

The first and last authors independently coded each response. Coding was conducted within a dataset that included only participant responses along with randomly generated participant numbers. To prevent bias, we lacked access to any other information about participants while coding, such as their age and parental incarceration status. Moreover, we developed coding guidelines prior to reviewing participants' responses. We did not discuss specific participant responses, or compare our codes, until after each person had independently coded all responses.

For each category, each coder assigned each response a 1 if it referenced the category and a 0 if it did not. A participant who described their parent as intelligent, angry, and temperamental received a 1 in the "positive" category due to the presence of at least one positive descriptor (intelligent), a 1 in the "negative" category due to the presence of at least one negative descriptor (in this case there were two, angry and temperamental), and a 0 in the "neutral" category. We did not initially include a "mixed" code for responses indicating both positive and negative descriptors; such responses simply received a 1 for both the "positive descriptor" and "negative descriptor" categories. Subsequently, we became aware that some past work testing children's views of their relationships with close others has included a separate "mixed" code (e.g., Johnson \& Easterling, 2015; Shlafer \& Poehlmann, 2010). However, such responses were rare in the present study. Only one child of an incarcerated parent and three children whose parents were not incarcerated provided "mixed" descriptions. For instance, one such child described his parent as being "nice" (a positive descriptor), but also being "angry at times" (a negative descriptor). Due to their overall rarity, the Main Text does not further discuss "mixed" responses to the open-ended question. See Supplementary Materials for descriptive statistics regarding the number of positive, negative, and neutral descriptions children provided when discussing their parents.

The raters achieved nearly perfect agreement when coding positive descriptors $(\kappa=.89)$ and moderate agreement when coding neutral descriptors $(\kappa=.45)$. Reliability was lower for negative descriptors $(\kappa=.27)$; however, this value likely indicates the low overall incidence 
of negative descriptors rather than low agreement among coders, as coders agreed that $87 \%$ of responses did not include negative descriptors. ${ }^{2}$ Nevertheless, caution is warranted when interpreting results concerning negative descriptors. Disagreements between coders were resolved via discussion.

\section{Results}

We used a Bonferroni correction to adjust analyses that included multiple comparisons. Below, we report the corrected alpha level alongside unadjusted $p$ values. Additionally, we report the smallest detectable effect size given the present samples. For ease of interpretation, we report both the effect sizes and their corresponding benchmark labels ("small", "medium", "large", Cohen, 1988); these sensitivity analyses were conducted assuming power $=.80$ and alpha $=.05$. Prior to conducting the primary analyses, we examined correlations between participants' responses and demographic factors such as age, length of parent-child separation, and frequency of parent-child contact. These factors did not reliably predict participants' responses. For brevity, we only report the correlations between participant responses and age in the Main Text; all other correlations are reported in Supplementary Materials.

\section{Open-ended descriptions of parents}

Children whose parents were not incarcerated largely described their parents using positive and neutral descriptors. Sixty-eight percent of children whose parents were not incarcerated described their parent using at least one positive descriptor, and $65 \%$ of children in this group described their parent using at least one neutral descriptor. Only $6 \%$ of children whose parents were not incarcerated spontaneously described their parent using a negative descriptor. A similar pattern of results emerged among children of incarcerated parents. Seventy-one percent of children described their incarcerated parents using at least one positive descriptor, and $63 \%$ of children in this group described their parent using at least one neutral descriptor. Only $8 \%$ of children spontaneously described their incarcerated parent using a negative descriptor (see Figure 1).

In addition to the descriptive statistics reported above, we correlated age with responses in each of the coding categories developed for examining children's descriptions of their parents. This approach resulted in three analyses among children of incarcerated parents and, separately, three analyses among children whose parents were not incarcerated, for a total of six correlations. Therefore, $p$ values needed to be .008 or lower to reach significance, and none did so $(|r| s \leq .32, p s \geq .028)$.

In summary, two main patterns emerged from children's open-ended descriptions of their parents. First, a higher percentage of children with non-incarcerated parents spontaneously described their parent using positive terms rather than negative terms. A similar pattern emerged among children of incarcerated parents. Second, the percentage of children

\footnotetext{
${ }^{2}$ Kappa is affected by the rarity of the finding being considered (Feinstein \& Cicchetti, 1990). For rare findings, low kappas may not signify low inter-coder agreement. This is the case in the present study: whereas the kappa for the "negative description" code was low, the coders agreed that $87.21 \%$ of responses did not include a "negative description" code. Because kappa does not differentiate among various types of disagreement, it is important to highlight that the coders agreed that negative descriptions were largely absent from participants' responses.
} 


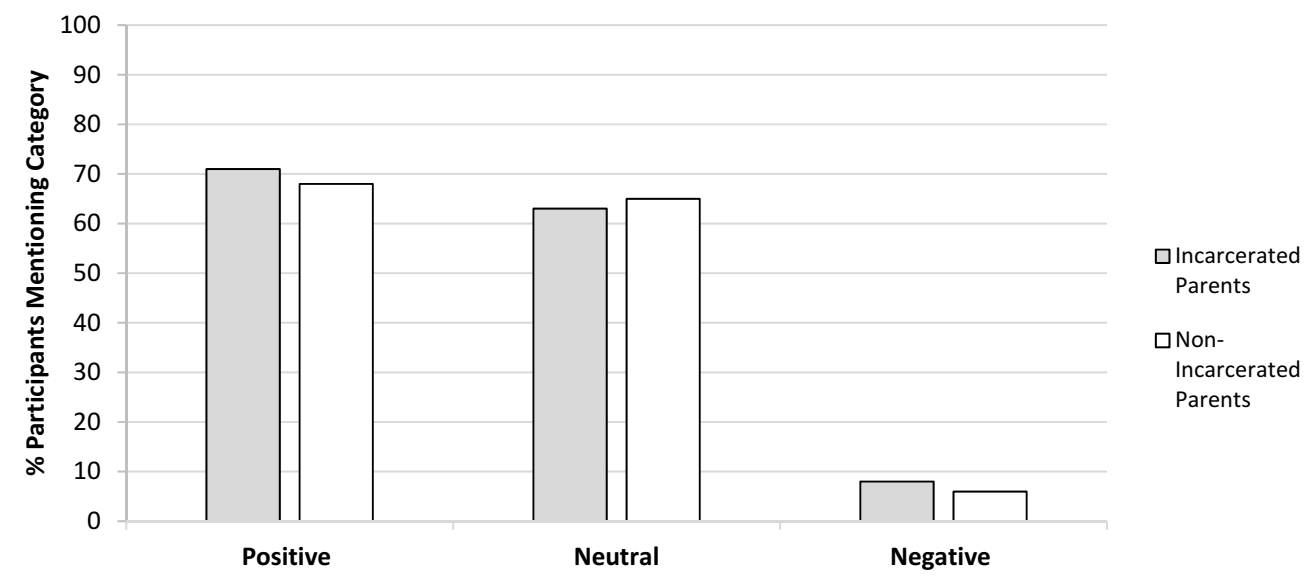

Figure 1. Percentage of participants using positive, neutral, and negative descriptions of their parent.

using positive, neutral, and negative descriptors was similar across groups. These descriptive results provide preliminary evidence that parental incarceration may not attenuate children's positivity toward their parents.

\section{Closed-ended reports of emotions when thinking about parents and friends}

Next, we analyzed the extent to which participants agreed feeling positive and negative emotions toward close others using a 2 (Participant Group: incarcerated parent vs. nonincarcerated parent) x 2 (Emotion Valence: positive vs. negative) x 2 (Target: parent vs. friend) mixed ANOVA with repeated measures on the last two factors. This analysis revealed main effects of Participant Group $\left(F(1,80)=5.97, p=.017, \eta_{\mathrm{p}}{ }^{2}=.07\right)$ and Emotion Valence $\left(F(1,80)=607.55, p<.001, \eta_{\mathrm{p}}{ }^{2}=.88\right)$, which were qualified by a Participant Group x Emotion Valence interaction $\left(F(1,80)=4.08, p=.047, \eta_{\mathrm{p}}{ }^{2}=.05\right)$. No other main effects or interactions reached significance ( $p s \geq .114)$. Given the nonsignificant effect of Target, we collapsed across items regarding parents and friends in all subsequent analyses. In other words, participants' agreement with items probing their emotions when thinking about close others served as the main dependent measure. Items measuring each type of emotion had acceptable reliability $\left(\alpha_{\text {positive }}=.69, \alpha_{\text {negative }}=.70\right)$.

To examine the Participant Group x Emotion Valence interaction, we conducted two sets of tests (see Figure 2). First, we investigated whether children of incarcerated parents reported differential agreement with statements referencing positive, versus negative, emotions when thinking about close others. We also conducted this analysis among children whose parents were not incarcerated. This analysis included two comparisons; therefore, $p$ values needed to be .025 or lower to pass the Bonferroni-corrected significance threshold. The sample size of children whose parents were not incarcerated allowed for detection of a "small" effect (Cohen's $d=.37$ ), and the sample size of children of incarcerated parents allowed for detection of a "medium" effect (Cohen's $d=.60$ ). All statistically significant pairwise comparisons yielded "large" effect sizes above these thresholds. Children of incarcerated parents were more likely to agree that thinking about close others made them feel positive emotions than they were to agree that thinking about close others 


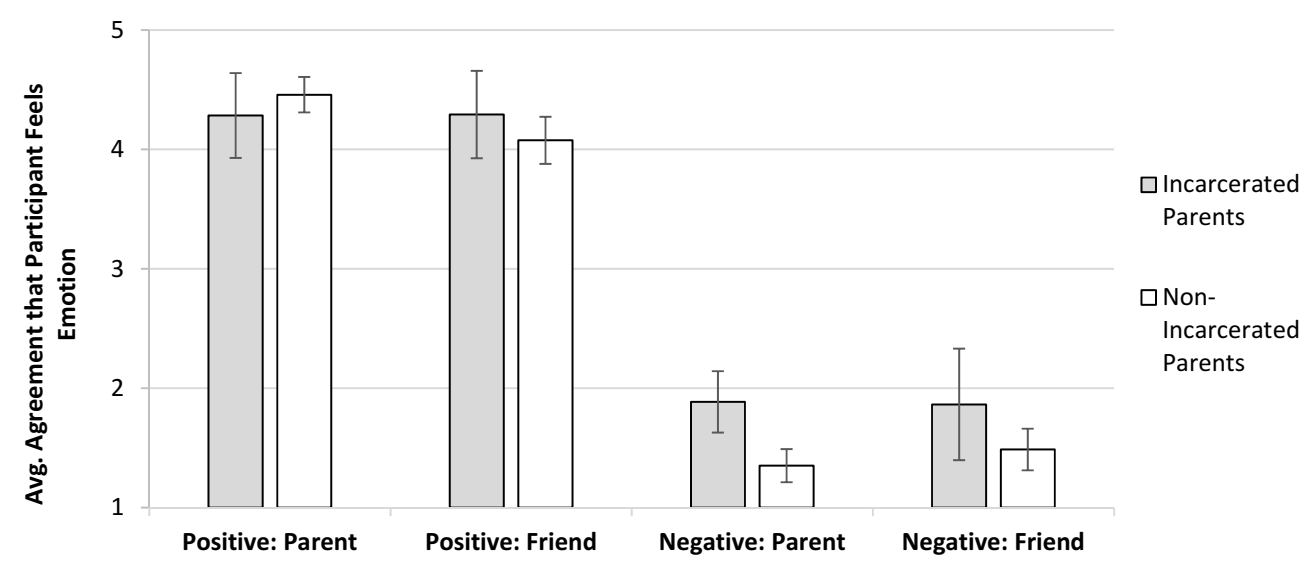

Figure 2. Average agreement with statements regarding feelings toward different important people in participants' lives. Higher values reflect higher levels of agreement that a given person elicits a specific emotion. Error bars represent $95 \%$ confidence intervals.

made them feel negative emotions ( $p<.001$, Cohen's $d=2.15,95 \%$ CI: $[2.61,3.07]$ ). This pattern of results also emerged among children whose parents were not incarcerated $(p<.001$, Cohen's $d=3.75,95 \%$ CI: $[2.06,2.77])$.

Second, we compared the responses of children with incarcerated parents with the responses of children whose parents were not incarcerated within each emotion category. This analysis included two comparisons; therefore, $p$ values needed to be .025 or lower to pass the Bonferroni-corrected significance threshold. A sensitivity analysis revealed that these analyses could detect "medium" effects (Cohen's $d=.69$ ). Children of incarcerated parents were more likely to agree that thinking about close others made them feel negative emotions than were children whose parents were not incarcerated $(p=.002$, Cohen's $d=.76,95 \%$ CI $[.17, .74])$. This pairwise comparison yielded a "medium" effect size above the threshold generated by the sensitivity analysis reported above. However, children of incarcerated and non-incarcerated parents did not significantly differ from each other when indicating the extent to which thinking about close others made them feel positive emotions $(p=.879$, Cohen's $d=.04,95 \%$ CI $[-.26, .31])$. To obtain more nuanced information about the null effect, we conducted a "multiverse" analysis in which we compared children of incarcerated parents and children whose parents were not incarcerated using two additional approaches: (a) Bayesian analyses (Dienes, 2014) and (b) equivalence testing (Lakens, 2017). These analyses suggested that the two groups of children did not substantially differ from each other in reports of positive emotion. More details about these analyses are presented in Supplementary Materials.

In addition to the primary analyses reported above, we examined the extent to which participants' age predicted their agreement with feeling positive and, separately, negative emotions when thinking about their parent. We also examined the extent to which participants' age predicted their agreement with feeling positive and, separately, negative emotions when thinking about their best friend. This approach resulted in four analyses among children of incarcerated parents and, separately, four analyses among children whose parents were not incarcerated, for a total of eight correlations. Therefore, $p$ values needed to be .008 or lower to reach significance, and none did so $(|r| s \leq .41, p s \geq .049)$. 


\section{Discussion}

The current work examined how parental incarceration might shape children's views of close others. Cultural messages in the United States propagate negative views of incarcerated people (Kleinfeld, 2016), and such messages could influence children's perspectives (e.g., Gelman, 2009). Further, children often express negativity toward transgressors, especially those who commit moral transgressions (Hardecker et al., 2016). Thus, it was possible that societal messages and children's own sensitivity to perceived moral transgression would lead them to express negativity when describing and thinking about their incarcerated parent. However, work highlighting the robustness of children's positivity (e.g., Boseovski \& Lee, 2006; Diesendruck \& Lindenbaum, 2009) led to an alternative prediction: that children would report positive views of their incarcerated parent. Consistent with the latter possibility, children of incarcerated parents more often described their parent using positive terms than negative terms. Moreover, children of incarcerated parents were more likely to agree that thinking about close others (their parent, their best friend) made them feel positive emotions than they were to agree that thinking about close others made them feel negative emotions.

As previously mentioned, it is possible that children's optimism and resiliency underlies their positivity when thinking about close others, including their incarcerated parents. However, an alternative explanation for this positivity exists: children may have reasoned that their parents did not commit a moral transgression at all. Instead, children may have inferred that societal reasons (e.g., racism, poverty) played a role in their parent's incarceration. At first blush, this possibility seems tenable. Societal inequality - particularly racism is a potent driver of incarceration in the United States (e.g., Alexander, 2012; Forbes, 2016; Forman, 2017; Harcourt, 2007). Police officers are more likely to surveil, use excessive force against, and arrest Black people compared to White people (e.g., Alexander, 2012; Forman, 2017; Harcourt, 2007; Joseph \& Pearson, 2002; Weitzer, 2015). Furthermore, Black people constitute $13 \%$ of the United States population but $40 \%$ of the population of people who are incarcerated in the United States (Sawyer \& Wagner, 2020). Given the link between societal factors (e.g., racism) and incarceration, it is possible that children of incarcerated parents attribute their parent's experiences to societal inequality or other forms of social injustice as opposed to their parent's moral transgression. In turn, such perspectives could attenuate children's negativity toward their incarcerated parents; future work can test this possibility.

Related to this possibility, a recent study (Dunlea \& Heiphetz, 2020) examined the extent to which parental incarceration shapes children's judgments about why people come in contact with the justice system. Children of incarcerated parents, like children whose parents were not incarcerated, readily attributed contact with the justice system to individual-level factors, such as a person's own behaviors (e.g., committing a crime) and their negative internal qualities (e.g., bad moral character, immoral desires). However, attributions referencing societal inequality (e.g., racism) were rare among children whose parents were not incarcerated and entirely absent among children of incarcerated parents. These results suggest that children of incarcerated parents may not readily attribute contact with the criminal justice system in general to societal inequality. Further, these results dovetail with a broader literature suggesting that people underestimate the extent to which negative life outcomes arise from societal inequality (e.g., Davidai \& Gilovich, 2015; Kraus, Rucker, \& Richeson, 2017). However, Dunlea and Heiphetz (2020) asked children to reason about 
people in general, not about their own incarcerated parent, and it is possible that children may form different judgments about their own mothers and fathers than about strangers. Future work can compare children's responses to questions about their incarcerated parents and, separately, incarcerated strangers to investigate this possibility.

In addition to the main finding described above, we also found that children of incarcerated parents were more likely to agree that thinking about close others made them feel negative emotions than were children whose parents were not incarcerated. What might underlie this negativity? One possibility is that children of incarcerated parents experience a greater number of childhood adversities than children whose parents are not incarcerated. In turn, this difference may explain the difference in reported negative emotion. This possibility is supported by work showing that children of incarcerated parents experience nearly five times more adverse events than children whose parents are not incarcerated (Turney, 2018). As previously mentioned, people who have experienced adversity in childhood are more likely than others to view the world - and those who inhabit it - in a negative light (Janoff-Bulman, 1989). Together, these lines of research may begin to elucidate why children of incarcerated parents were more likely than children of non-incarcerated parents to agree that thinking about their close others elicited negative emotions. Future research can directly test this possibility.

As well as providing insight into the role that parental incarceration may play in informing children's views of their parents, the current work makes two additional theoretical contributions. First, the present work clarifies the role of parental incarceration in shaping children's views of individuals with whom they have different types of close relationships. Past work showing that children readily differentiate perceived transgressors from non-transgressors (e.g., Yang et al., 2018) suggests that children may hold different views of their incarcerated parents and their friends. However, our work suggests that children of incarcerated parents view close others positively regardless of whether they are responding about their parent or friend. Second, the current data provide evidence that children's positivity toward close others - including their parents - supersedes the negativity associated with perceived transgression. In conjunction with research testing adults (e.g., Lee \& Holyoak, 2020), the present work suggests that positivity toward close others is an enduring component of social cognition. Future work can test children and adults in the same paradigm to obtain a more nuanced measure of age-related changes in positivity toward close others.

Alongside these theoretical contributions, understanding the experiences of children with incarcerated parents also has translational implications. The current work shows that this group of children are more likely to agree that thinking about close others makes them feel positive, versus negative, emotions. This finding could be linked with positive outcomes such as psychological resilience (Tugade \& Fredrickson, 2004). Debates have centered on whether it is beneficial for children to have contact with their incarcerated parents (Poehlmann, Dallaire, Loper, \& Shear, 2010), with some arguing that contact may be harmful because children may feel negative emotions if they were to engage with their incarcerated parents. Our data speak against this possibility, suggesting that such childparent relationships have a great deal of positivity.

While the present research brings to light the experiences of an underrepresented population (children of incarcerated parents) and provides critical insight into the role of parental incarceration in shaping social cognitive processes, several avenues are open for 
future research. Follow-up work can probe children's views of their non-incarcerated caregivers. Caregivers often form important relationships with children during a parent's incarceration, and positivity within these relationships can reduce the prevalence of negative outcomes typically associated with parental incarceration (Mackintosh, Myers, \& Kennon, 2006). Given the link between interpersonal emotions and developmental outcomes, elucidating children's emotions toward their caregivers may be important when considering interventions aimed at promoting positive developmental trajectories for children of incarcerated parents. The current work compared children's responses regarding an incarcerated parent versus a best friend because best friends are an important close relationship for children (Cleary et al., 2002), allowing us to ask all children in our study about a similar type of relationship. In contrast, great heterogeneity exists in caregiver identity: children of incarcerated parents may live with a non-incarcerated parent, grandparents, aunts and uncles, or foster families, among other possibilities (Hairston, 2009; Johnson \& Waldfogel, 2002). The current work lacked the power to compare these different types of relationships, but this remains an important avenue for future research.

Another question for future research concerns children's understanding of why their parents were incarcerated. We did not receive approval to probe children's knowledge about the reason behind their parent's incarceration; however, this factor may influence children's responses. Children typically expect individuals in close relationships to refrain from harming one another (Chalik \& Dunham, 2020) and respond especially negatively toward transgressors who harm familiar, versus unfamiliar, individuals (Abrams, Palmer, Rutland, Cameron, \& Van de Vyver, 2014). Thus, children who know that their parent is incarcerated for committing a crime against a close other (e.g., abusing the child's nonincarcerated parent) may be especially likely to report negativity when thinking about their incarcerated parent. Children who know that their parent is incarcerated for committing a crime against a distant other (e.g., a member of a social outgroup) or a crime without an immediate human victim (e.g., tax evasion) may report less negativity when thinking about their incarcerated parent. Future work can examine these possibilities.

Finally, future research can address a limitation concerning the dependent measure used in the present research. In the present study, children reported the extent to which they agreed that they felt certain emotions when thinking about close others. However, this approach is potentially limited because it only captures children's agreement that thinking about close others elicits positive and negative emotions rather than capturing children's experienced emotions. Nevertheless, this measure may be a reliable proxy for emotional experience. Past work suggests that people's thoughts influence their experienced emotions (for a review, see Ochsner \& Gross, 2004) and that people can explicitly report on the directional link between thought and experienced emotion (Davis, Levine, Lench, \& Quas, 2010; Lagattuta \& Wellman, 2001; Lagattuta, Wellman, \& Flavell, 1997). For example, 3- to 6-year-old children report that people would feel sad if they were to think of their pet rabbit that ran away (Lagattuta et al., 1997). In other words, children understand that thinking about a specific target (e.g., a parent, a rabbit) can influence experienced emotion (e.g., feeling happy, feeling sad). Davis et al. (2010) built on this past work by examining how 5and 6-year-old children describe strategies to change their emotions. Most children reported that they change their emotions by changing their thoughts. The authors interpreted this finding as evidence that children have a sophisticated understanding that their thoughts dictate their emotions. Extrapolating from this past research, children's emotions 
stemming from thinking about close others may correspond to their experienced emotions toward close others. Nevertheless, future work can seek to replicate the current findings by using additional measures that more directly measure experienced emotion.

\section{Conclusion}

We investigated the role of parental incarceration in shaping children's views of close others. Children of incarcerated parents more often described their parents using positive than negative terms. Moreover, children of incarcerated parents were more likely to agree that thinking about close others made them feel positive, versus negative, emotions. Similar patterns of results emerged among children whose parents were not incarcerated. In sum, the present work suggests that positivity when thinking about close others may be more powerful than the negativity surrounding perceived moral transgression.

\section{Acknowledgments}

The authors thank Riley Burke, Tamás Gömöri, Kaya Hantsbarger, Brendan Krovatin, Nina Mandracchia, Rachel Mulholland, Emily Nakkawita, Alexa Roman, Carolina Santiago-Robles, and Andre Senecal for assistance with data collection and coding; Andrea Fields, Megan Goldring, Dara Huggins, Christopher Medina-Kirchner, Federica Pinelli, Isaac Scott, and Fan Yang for their feedback; and our community partners for facilitating this research.

\section{Disclosure statement}

The authors do not have any potential conflict of interest.

\section{Funding}

This work has been supported (in part) by Columbia University and by a Visiting Scholar Award from the Russell Sage Foundation to LH. Any opinions expressed are those of the authors alone and should not be construed as representing the opinions of the Foundation.

\section{ORCID}

James P. Dunlea (D) http://orcid.org/0000-0003-2499-4970

\section{Data availability statement}

Because the data came from a vulnerable participant group and responses to our open-ended questions could potentially be used to identify participants, we are not able to post responses online. However, we are very happy to share the data individually with interested researchers; please contact [James Dunlea] at [james.dunlea@columbia.edu]. Please see the Appendix A for relevant study materials. 


\section{Open scholarship}

This article has earned the Center for Open Science badge for Open Materials. The materials are openly accessible at http://dx.doi.org/10.17632/9p44zzm595.2.

\section{References}

Abrams, D., Palmer, S. B., Rutland, A., Cameron, L., \& Van de Vyver, J. (2014). Evaluations of and reasoning about normative and deviant ingroup and outgroup members: Development of the black sheep effect. Developmental Psychology, 50(1), 258-270. doi:10.1037/a0032461

Alexander, M. (2012). The new Jim Crow: Mass incarceration in the age of colorblindness. New York, NY: The New Press.

Arditti, J. A. (2012). Parental incarceration and the family: Psychological and social effects of imprisonment on children, parents, and caregivers. New York: New York University Press.

Birnbaum, D., Deeb, I., Segall, G., Ben-Eliyahu, A., \& Diesendruck, G. (2010). The development of social essentialism: The case of Israeli children's inferences about Jews and Arabs. Child Development, 81(3), 757-777. doi:10.1111/j.1467-8624.2010.01432.x

Blaine, B. E., \& Brenchley, K. J. M. C. (2018). Understanding the psychology of diversity (3rd ed.). Thousand Oakes, CA: Sage.

Boseovski, J. J., \& Lee, K. (2006). Children's use of frequency information for trait categorization and behavioral prediction. Developmental Psychology, 42(3), 500-513. doi:10.1037/00121649.42.3.500

Cassidy, J. (1994). Emotion regulation: Influence of attachment relationships. Monographs of the Society for Research in Child Development, 59(2-3), 228-249. doi:10.2307/1166148

Chalik, L., \& Dunham, Y. (2020). Beliefs about moral obligation structure children's social categorybased expectations. Child Development, 91(1), e108-e119. doi:10.1111/cdev.13165

Cimpian, A., \& Markman, E. M. (2009). Information learned from generic language becomes central to children's biological concepts: Evidence from their open-ended explanations. Cognition, 113(1), 14-25. doi:10.1016/j.cognition.2009.07.004

Cleary, D. J., Ray, G. E., LoBello, S. G., \& Zachar, P. (2002). Children's perceptions of close peer relationships: Quality, congruence, and meta-perceptions. Child Study Journal, 32, 179-192.

Cohen, J. (1988). Statistical power analysis for the behavioral sciences (2nd ed.). Hillsdale, NJ: Erlbaum.

Davidai, S., \& Gilovich, T. (2015). Building a more mobile America-One income quintile at a time. Perspectives on Psychological Science, 10(1), 60-71. doi:10.1177/1745691614562005

Davis, E. L., Levine, L. J., Lench, H. C., \& Quas, J. A. (2010). Metacognitive emotion regulation: Children's awareness that changing thoughts and goals can alleviate negative emotions. Emotion, 10(4), 498-510. doi:10.1037/a0018428

Dienes, Z. (2014). Using Bayes to get the most out of non-significant results. Quantitative Psychology and Measurement, 5, 781. doi:10.3389/fpsyg.2014.00781

Diesendruck, G., \& Haber, L. (2009). God's categories: The effect of religiosity on children's teleological and essentialist beliefs about categories. Cognition, 110(1), 100-114. doi:10.1016/j. cognition.2008.11.001

Diesendruck, G., \& Lindenbaum, T. (2009). Self-protective optimism: Children's biased beliefs about the stability of traits. Social Development, 18(4), 946-961. doi:10.1111/j.1467-9507.2008.00494.x

Dunlea, J. P., \& Heiphetz, L. (2020). Children's and adults' understanding of punishment and the criminal justice system. Journal of Experimental Social Psychology, 87, 103913. doi: 10.1016/j. jesp.2019.103913

Feinstein, A. R., \& Cicchetti, D. V. (1990). High agreement but low kappa: I. The problems of two paradoxes. Journal of Clinical Epidemiology, 43(6), 543-549. doi:10.1016/0895-4356(90)90158-L 
Forbes, F. A. (2016). Invisible men: A contemporary slave narrative in the era of mass incarceration. New York, NY: Skyhorse Publishing.

Forman, J., Jr. (2017). Locking up our own: Crime and punishment in Black America. New York, NY: Farrar, Straus and Giroux.

Ganea, P. A., Lillard, A. S., \& Turkheimer, E. (2004). Preschoolers understanding of the role of mental states and action in pretense. Journal of Cognition and Development, 5(2), 213-238. doi:10.1207/ s15327647jcd0502_3

Gelman, S. A. (2009). Learning from others: Children's construction of concepts. Annual Review of Psychology, 60(1), 115-140. doi:10.1146/annurev.psych.59.103006.093659

Gerhardt, S. (2004). Why love matters: How affection shapes a baby's brain. London, UK: BrunnerRoutledge.

Hairston, C. F. (2009). Kinship care when parents are incarcerated: What we know, what we can do. Retrieved from https://folio.iupui.edu/bitstream/handle/10244/402/10147801_Kinship_Paper06a \%25203. pdf?sequence $=2$

Harcourt, B. E. (2007). Against prediction: Profiling, policing, and punishing in an actuarial age. Chicago, IL: The University of Chicago Press.

Hardecker, S., Schmidt, M. F., Roden, M., \& Tomasello, M. (2016). Young children's behavioral and emotional responses to different social norm violations. Journal of Experimental Child Psychology, 150, 364-379. doi:10.1016/j.jecp.2016.06.012

Janoff-Bulman, R. (1989). Assumptive worlds and the stress of traumatic events: Applications of the schema construct. Social Cognition, 7(2), 113-136. doi:10.1521/soco.1989.7.2.113

Jeffreys, H. (1961). Theory of probability (3rd ed.). Oxford, England: Oxford University Press.

Johnson, E. I., \& Easterling, B. A. (2015). Coping with confinement: Adolescents' experiences with parental incarceration. Journal of Adolescent Research, 30(2), 244-267. doi:10.1177/ 0743558414558593

Johnson, E. I., \& Waldfogel, J. (2002). Parental incarceration: Recent trends and implications for child welfare. Social Service Review, 76(3), 460-479. doi:10.1086/341184

Joseph, J., \& Pearson, P. G. (2002). Black youths and illegal drugs. Journal of Black Studies, 32(4), 422-438. doi:10.1177/002193470203200404

Kleinfeld, J. (2016). Two cultures of punishment. Stanford Law Review, 68, 933-1036.

Kraus, M. W., Rucker, J. M., \& Richeson, J. A. (2017). Americans misperceive racial economic equality. Proceedings of the National Academy of Sciences, 114, 10324-10331. doi: 10.1073/ pnas.1707719114

Lagattuta, K. H., \& Wellman, H. M. (2001). Thinking about the past: Early knowledge about links between prior experience, thinking, and emotion. Child Development, 72(1), 82-102. doi:10.1111/ $1467-8624.00267$

Lagattuta, K. H., Wellman, H. M., \& Flavell, J. H. (1997). Preschoolers' understanding of the link between thinking and feeling: Cognitive cuing and emotional change. Child Development, 68(6), 1081-1104. doi:10.1111/j.1467-8624.1997.tb01986.x

Lakens, D. (2017). Equivalence tests: A practical primer for $t$ tests, correlations, and meta- analyses. Social Psychological and Personality Science, 8(4), 355-362. doi:10.1177/1948550617697177

Lakens, D., \& Evers, E. R. K. (2014). Sailing from the seas of chaos into the corridor of stability: Practical recommendations to increase the informational value of studies. Perspectives on Psychological Science, 9(3), 278-292. doi:10.1177/1745691614528520

Lee, J., \& Holyoak, K. J. (2020). "But he's my brother": How family obligation impacts moral judgments. Memory \& Cognition, 48(1), 158-170. doi: 10.3758/s13421-019-00969-7

Mackintosh, V. H., Myers, B. J., \& Kennon, S. S. (2006). Children of incarcerated mothers and their caregivers: Factors affecting the quality of their relationship. Journal of Child and Family Studies, 15 (5), 579-596. doi:10.1007/s10826-006-9030-4

Miller, K. M. (2006). The impact of parental incarceration on children: An emerging need for effective interventions. Child and Adolescent Social Work Journal, 23(4), 472-486. doi:10.1007/s10560-0060065-6

Mumola, C. J. (2000). Incarcerated parents and their children. Retrieved from https://www.bjs.gov/ content/pub/pdf/iptc.pdf 
Murphey, D., \& Cooper, P. M. (2015). Parents behind bars: What happens to their children? Retrieved from http://www.courts.ca.gov/documents/BTB_23_4K_6.pdf

Murray, J., \& Farrington, D. P. (2008). The effects of parental imprisonment on children. Crime and Justice, 37(1), 133-206. doi:10.1086/520070

Nesmith, A., \& Ruhland, E. (2008). Children of incarcerated parents: Challenges and resiliency, in their own words. Children and Youth Services Review, 30(10), 1119-1130. doi:10.1016/j. childyouth.2008.02.006

Ochsner, K. N., \& Gross, J. J. (2004). Thinking makes it so: A social cognitive neuroscience approach to emotion regulation. In R. F. Baumeister \& K. D. Vohs (Eds.), Handbook of selfregulation: Research, theory, and applications (pp. 229-255). Erlbaum, NJ: The Guilford Press.

Olson, K. R., Key, A. C., \& Eaton, N. R. (2015). Gender cognition in transgender children. Psychological Science, 26(4), 467-474. doi:10.1177/0956797614568156

Poehlmann, J., Dallaire, D., Loper, A. B., \& Shear, L. D. (2010). Children's contact with their incarcerated parents: Research findings and recommendations. American Psychologist, 65(6), 575-598. doi:10.1037/a0020279

Rhodes, M. (2014). Children's explanations as a window into their intuitive theories of the social world. Cognitive Science, 38(8), 1687-1697. doi:10.1111/cogs.12129

Rouder, J. N., Speckman, P. L., Sun, D., Morey, R. D., \& Iverson, G. (2009). Bayesian $t$ tests for accepting and rejecting the null hypothesis. Psychonomic Bulletin \& Review, 16(2), 225-237. doi:10.3758/PBR.16.2.225

Sawyer, W., \& Wagner, P. (2020). Mass incarceration: The whole pie 2020. Retrieved from https:// www.prisonpolicy.org/reports/pie2020.html

Shlafer, R. J., \& Poehlmann, J. (2010). Attachment and caregiving relationships in families affected by parental incarceration. Attachment \& Human Development, 12(4), 395-415. doi:10.1080/ 14616730903417052

Simmons, J., Nelson, L., \& Simonsohn, U. (2013). Life after p-hacking. Paper presented at the annual meeting of the Society for Personality and Social Psychology. New Orleans, LA.

Tasimi, A., \& Johnson, M. K. (2019). Children's initial responses and beyond: Effects of niceness and similarity on preference, giving, and memory. Child Development, 90(2), 432-440. doi:10.1111/ cdev.13196

Tugade, M. M., \& Fredrickson, B. L. (2004). Resilient individuals use positive emotions to bounce back from negative emotional experiences. Journal of Personality and Social Psychology, 86(2), 320-333. doi:10.1037/0022-3514.86.2.320

Turney, K. (2018). Adverse childhood experiences among children of incarcerated parents. Children and Youth Services Review, 89, 218-225. doi:10.1016/j.childyouth.2018.04.033

Watson, D., Clark, L. A., \& Tellegen, A. (1988). Development and validation of brief measures of positive and negative affect: The PANAS scales. Journal of Personality and Social Psychology, 54(6), 1063-1070. doi:10.1037/0022-3514.54.6.1063

Weidman, A. C., Sowden, W. J., Berg, M. K., \& Kross, E. (2019). Punish or protect? How close relationships shape responses to moral violations. Personality and Social Psychology Bulletin. doi:10.1177/0146167219873485

Weitzer, R. (2015). American policing under fire: Misconduct and reform. Society, 52(5), 475-480. doi:10.1007/s12115-015-9931-1

Yang, F., Choi, Y. J., Misch, A., Yang, X., \& Dunham, Y. (2018). In defense of the commons: Young children negatively evaluate and sanction free riders. Psychological Science, 29(10), 1598-1611. doi:10.1177/0956797618779061

Zimmer-Gembeck, M. J., \& Skinner, E. A. (2011). The development of coping across childhood and adolescence: An integrative review and critique of research. International Journal of Behavioral Development, 35(1), 1-17. doi:10.1177/0165025410384923 


\section{Appendix A. Research materials}

The below document includes only instructions and items relevant to the research questions posed in the main text. During each experimental session, participants responded to additional items that were part of a different project probing children's understanding of punishment and the criminal justice system. Participants completed one of three possible versions of this study; for brevity, we include only one of the versions below. The order in which participants answered questions about their parent or best friend and the order in which the closed-ended items were presented were counterbalanced across versions.

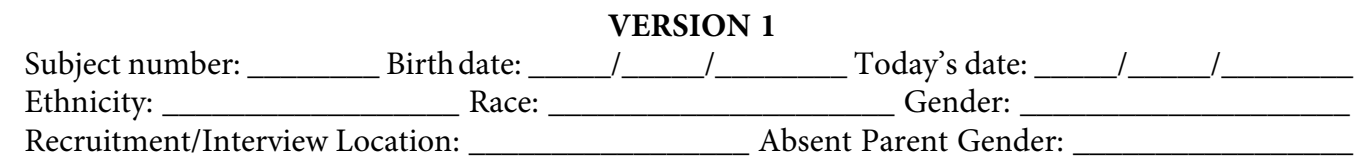

Start by asking the child how many years old they are and note this answer in the space below. Then read the appropriate assent script from the front of the binder. If child says he/she wants to do the study, continue with the questions below.

How old are you? figuring out which assent script to read) years (fill this in based on info you get when

When is your birthday? $\mathrm{MM} / \mathrm{DD}$, e.g., $01 / 20$

Are you a boy or a girl?

\section{SECTION 1}

First, I'm just curious to learn a little more about your mom/dad. What is she/he like? [write everything child says]

Great, thank you so much for sharing that with me. Do you want to keep going?

[If yes, proceed; if no, end study.]

\section{SECTION 2}

For the next few questions, I'm going to tell you something, and I want you to tell me how much you agree or disagree with the thing I just said. To do that, we're going to use these pictures over here. [Point to stick figure scale.] If you don't agree at all, you would point here [point to smallest person]. If you agree a little bit, you would point here [point to second-smallest person]. If you agree a medium amount, you would point here [point to middle person]. If you agree a lot, you would point here [point to second-largest person]. And if you agree completely, you would point here [point to biggest person]. Does that make sense?

Okay, here's my first sentence: Right now, we're in the United States of America. How much do you agree with that?

Christmas is in the summer. How much do you agree with that?

How much do you agree that thinking about your mom/dad makes you feel ... proud angry loved happy excited scared calm nervous 
sad

How much do you agree that thinking about your best friend makes you feel ...

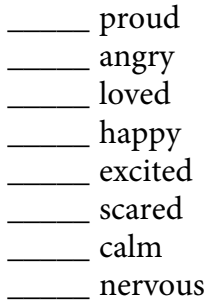

nervous

sad

Thank you so much for answering all of those questions for me. You did a great job! I'm trying to learn about how kids think about other people, and talking with you helped me a lot. Like I said when we started, none of the questions had any right or wrong answers, and it was very helpful to me to hear your thoughts in response to these questions.

\section{Appendix B. Likert-type scale used for closed-ended items}
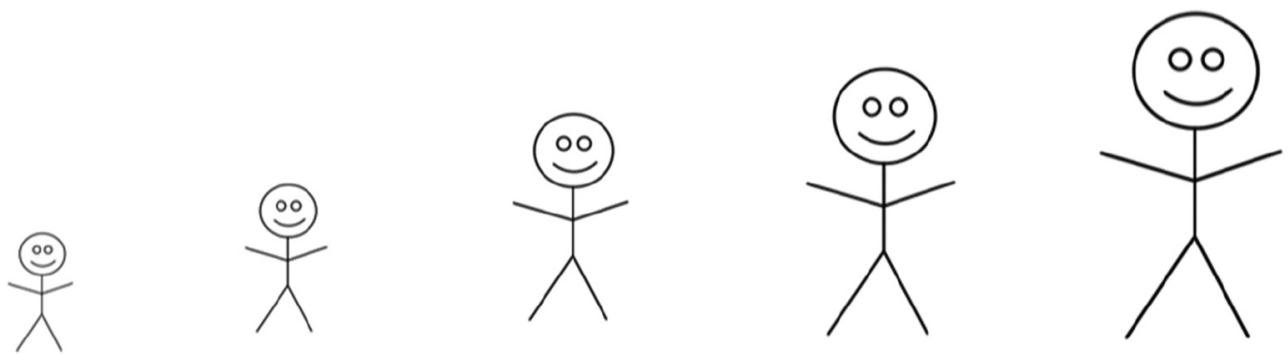